\title{
Virtual Marine Education Environment Creation Method
}

\author{
Wu Jinlong ${ }^{\mathrm{a}}$, Zhang Rukai ${ }^{\mathrm{b}}$ \\ Tianjin Maritime College, No. 8, Yashen Road, Haihe Education Park, Tianjin \\ 4443950743@qq.com, bkaidesky@163.com
}

Keywords: Multigen Creator, Unity 3D, Virtual Marine

\begin{abstract}
Multigen Creator will be used to model, and after format conversion, the model will be driven by Unity 3D to complete the virtual ship platform construction. By introducing control logic such as roaming logic and navigation logic, the user is allowed to perform operations such as roaming and navigation in the virtual scene. The system has the characteristics of strong sense of reality, friendly interface, and interaction, which meets the needs of information teaching. With the cross-platform features of Unity3d, PC and mobile programming are respectively carried out to realize the network development of VR training platform.
\end{abstract}

\section{Introduction}

Virtual Reality (VR) is a scientific method and science that is created and produced by human beings in the process of exploring nature and understanding nature. It is gradually formed to understand nature and simulate nature, and then better adapt and utilize nature. Using virtual reality technology, based on real scenes, relying on geographic information technology, 3D modeling technology and game engine to construct a virtual reality scene with both realism and interactivity.

In recent years, the informatization construction of the campus has been rapidly developed, and the development of virtual teaching resources is an important part of the campus information construction. Most of the traditional digital teaching resources are two-dimensional data, which is difficult to meet the needs of contemporary information teaching [1]. The latest virtual reality technology has the characteristics of strong sense of reality, interaction, multi-device compatibility, etc., and its application in the construction of information resources can make up for the inherent deficiencies of traditional teaching resources.

In view of the above background, this paper uses virtual reality technology, uses Multigen Creator to build 3D models, builds virtual scenes with Unity3D, and introduces corresponding control logic through C\# programming to construct a virtual ship with both realism and interactivity $[2,3]$. The virtual ship supports operations such as roaming and navigation, and can be deployed on different platforms to provide services for campus publicity, planning, and information management [4]. 


\section{System design}

\subsection{Building process}

In the process of system development, based on the software engineering methodology, the functions of each module in the system are designed based on actual needs. The development process of the system is mainly divided into three parts: model construction, 3D virtual scene construction and introduction of control logic. In addition, the developed system can be deployed on different platforms with cross-platform compatibility.

The construction method of model construction 3D model is becoming more and more mature.

Multigen creator is a software package developed by Multigen-Paradigm of the United States to create real-time 3D models for visual simulation. Efficient and optimal generation of real-time three-dimensional (RT3D) database, and closely integrated with subsequent real-time simulation software, is conducive to saving graphics workstation rendering overhead, ensuring system fluency and multi-terminal sharing experience.

After obtaining the ship's frame through data import in the early stage, the ship's structural details and the size of the equipment entity can be obtained through field measurement[5]. The ship's structural details and equipment entities should be as close as possible to the actual ship, in the position where the details need to be reflected (eg, the compass light) The position can be adjusted as needed.

In recent years, Unity Technologies has developed the Unity graphics and image engine to make building visualization and real-time 3D animation easy to create. After the model is built, simply import it into Unity3D and visually complete the layout of the objects in the scene to complete the construction of the 3D virtual scene[6]. To enhance the realism of the virtual scene, further configuration of the objects in the scene is required. For example, in the real world, objects are not allowed to walk freely. According to this rule, a collision detection mechanism needs to be added to objects in the virtual scene to avoid the phenomenon of passing between rigid bodies.

Unity supports writing control logic in C\# and JavaScript. From the functional division, the control logic can be divided into: roaming logic (which defines the roaming behavior of the characters in the system, such as the way of roaming, the speed of roaming, etc.), navigation logic (the navigation method of the character), and interaction logic (mainly including users and Interface interaction, role interaction with objects within the system, etc[7].

\subsection{Functional Analysis}

\subsubsection{Manuall roaming}

Manual roaming manipulates the movement of characters within the system through control information from external devices. In the virtual scene, you can use the keyboard's "W", "A", "S", "D" keys to control the movement of the character, press the right mouse button and drag to rotate the view angle, press the space bar to jump. During the roaming process, the character encounters obstacles and stops moving forward. This system uses the first person character control preset body that comes with Unity3D, which defines the movement, jumping, and acceleration of the character when the character moves, making the character's movement process realistic.

\subsubsection{Automatic roaming}

Auto roaming allows a character to roam along a given route without any input after the roaming event is triggered. The auto-roaming feature can be triggered by components of the user interface layer, and the character will automatically move according to the preset path[8]. The 
implementation of the automatic roaming function is realized by the external plug-in-behavior tree in Unity3D, which is mainly applied to the AI behavior control of the objects in the system, and the control of the role can be realized by the combination of the nodes on the behavior tree.

\subsubsection{Virtual navigation}

Virtual navigation refers to the input of the current coordinate point of the character and the target point. The corresponding navigation method is used to generate the route from the starting point to the target point. This function is also implemented through the behavior tree. Unlike automatic roaming, virtual navigation first has to be baked out of the scene's navigation grid through Unity3D. In order to simplify the operation and lower the usage threshold, a static map can be generated in advance in the scene, and the external user only needs to visually select the target position on the map to navigate.

\section{System implementation and testing based on the above construction method}

\subsection{Ship hull model}

It can realize the overall display of the ship, facilitate the analysis of the hull structure and the division of the ship area, and facilitate the macroscopic understanding of the ship. At the same time, it is the development platform for other functional virtual devices, as well as the working basis and behavior carrier for in-depth development of ship survival, ship fire, boat operation, anchoring, cargo operation [9].

The main place where the bridge is working as a deck crew is the installation and storage of internal communication equipment, external communication equipment, navigation equipment, distress warning equipment, meteorological equipment, control equipment connected to the engine room and other necessary equipment. The space is relatively small. And the relevant equipment must be placed in accordance with relevant regulations. The VR laboratory can describe the resettlement of related facilities and equipment in a flexible way, which can directly reflect the impact of each ship structure on the facilities and equipment, and provide a unique perspective that can not be achieved by actual ship observation.

\subsection{Propeller work simulation}

The propeller is under water, and it is not easy to apply the real ship propeller working scene to teaching. The existing related resources are mainly obtained through animation simulation or real ship shooting. The two forms of teaching resources can't achieve all-round multi-angle observation. At the same time, the video effect will also affect its clarity due to the underwater environment. The hull-propeller-rudder attachment relationship can be presented using virtual modeling techniques. With Unity3D's Particle System, you can realize the dynamic flow performance of the ship's tail water flow under the joint action of the hull-propeller-rudder. It can be used to vividly describe the working principle of the rudder, the propeller biasing effect and the ship's directionality to the ship. Sexual influences are difficult to understand $[10,11]$. 


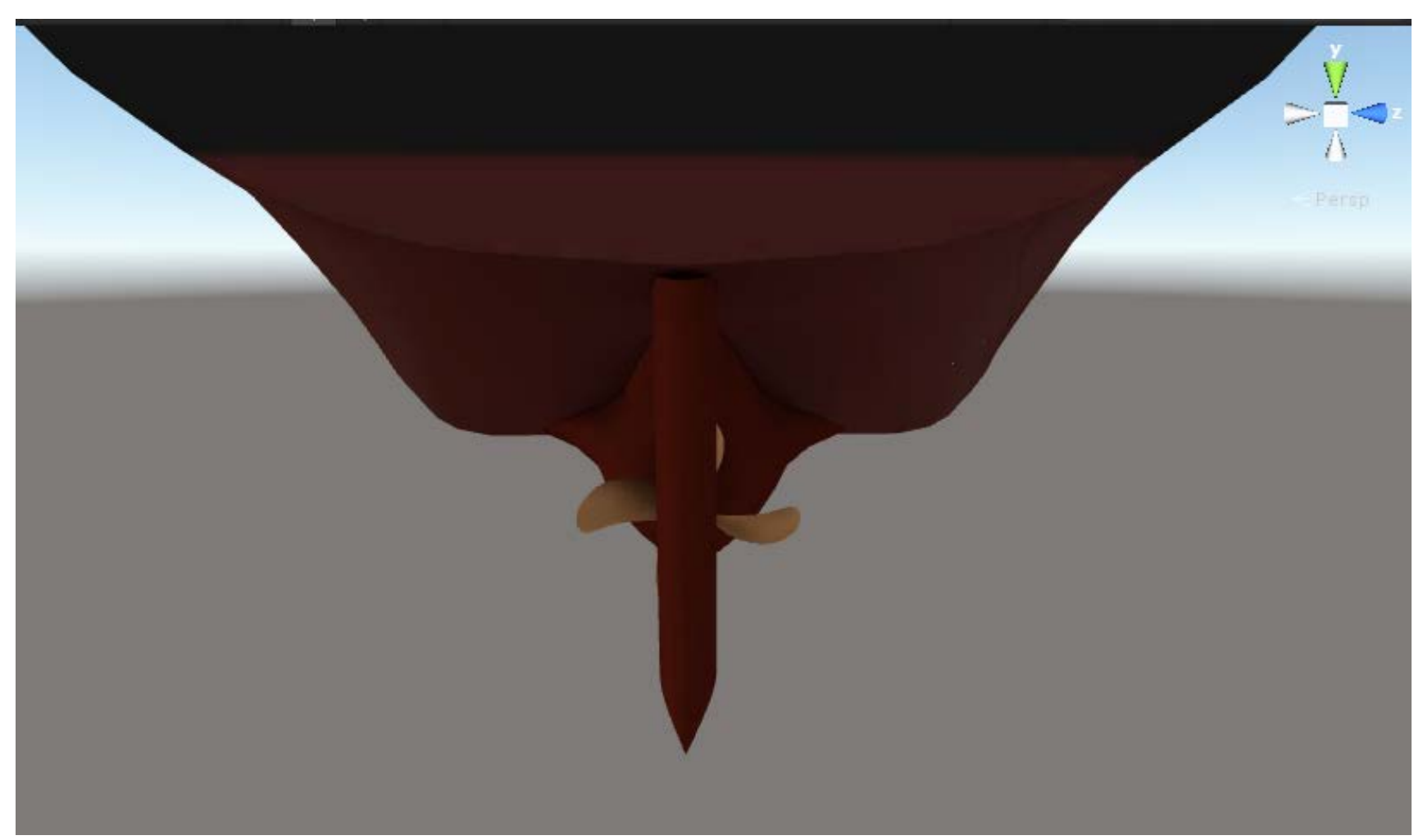

Figure 1. Virtual ship exterior map.

\section{Conclusion}

The virtual ship based on the design method of this paper uses Multigen Creator to build the model and build with the Unity3D 3D virtual scene. In addition, the introduction of control logic solves the problem of interaction between external users and virtual scenes. The virtual reality interactive system built is realistic and practical. However, this method still has certain limitations. For example, the system has relatively single functions and the data does not have persistence. In future research, rich interaction logic and database connection to achieve dynamic access of data will be the focus.

\section{Acknowledgments}

This work was financially supported by Tianjin City Jinnan District Municipal Science\& Technology Commission science and technology planning projects (20171508).

\section{References}

[1] Hu Xianmin. Application of Virtual Reality Technology in Classroom Teaching Reform for Navigation Majors. Journal of Zhejiang Institute of Communications, 2016(4): 49-52.

[2] Zhang Gutai, Zhu Wenqiu, Zhang xiaoyun. Virtual laboratory simulation based on Unity3D. China Computer \& Communication, 2017(3): 30-32.

[3] Zhu huijuan. Virtual Roaming System Based on Unity3D. Computer Systems \& Applications, 2012,21(10):36-39.

[4] AbdullahNAS, RusliNIA, IbrahimMF. Mobile game size estimation: COSMICFSM rules, UML mapping model and Unity3D game engine // OpenSystems. IEEE, 2015: 42-47.

[5] Zhu H J. Virtual Roaming System Based on Unity3D. Computer Systems \& Applications, 2012.

[6] Xie J. Research on key technologies base Unity3D game engine// International Conference on Computer Science \& Education. IEEE, 2012: 695-699.

[7] Yang H Z, Kang F J, Chu Y J, et al. Random Wave Simulation and Validation Based on Ocean Wave Spectrum [J]. 
Acta Simulata Systematica Sinica, 2005.

[8] Jia X, Tian W, Yang Y. Chemical Accident Evolutionary Scenario Study Based on Unity3D [J]. Journal of Qingdao University of Science \& Technology, 2017.

[9] He haiyang. Practice in the Optimal Layout of Ship's Cabin. Ship \& Ocean Engineering, 2007, 36(4):12-14.

[10] Lu GP, Xue GH, Chen Z. Design and Implementation of Virtual Interactive Scene Based on Unity3D. Advanced Materials Research, 2011, 317-319:2162-2167.

[11] Bouws E, Günther H, Rosenthal W, et al. Similarity of the wind wave spectrum in finite depth water: 1 . Spectral form [J]. Journal of Geophysical Research Oceans, 1985, 90(C1):975-986. 